\title{
Studying the Benefits and Challenges of Spatial Distribution and Physical Affordances in a Multi-Device Workspace
}

\author{
Johannes Zagermann, Ulrike Pfeil, Carmela Acevedo, and Harald Reiterer \\ HCI Group, University of Konstanz,
}

\{johannes.zagermann,ulrike.pfeil,harald.reiterer\}@uni-konstanz.de, carmela.acevedo@gmail.com

\begin{abstract}
In recent years, research on cross-device interaction has become a popular topic in HCI leading to novel interaction techniques mutually interfering with new evolving theoretical paradigms. Building on previous research, we implemented an individual multi-device work environment for creative activities. In a study with 20 participants, we compared a traditional toolbar-based condition with two conditions facilitating spatially distributed tools on digital panels and on physical devices. We analyze participants' interactions with the tools, encountered problems and corresponding solutions, as well as subjective task load and user experience. Our findings show that the spatial distribution of tools indeed offers advantages, but also elicits new problems, that can partly be leveraged by the physical affordances of mobile devices.
\end{abstract}

\section{ACM Classification Keywords}

H.5.m. Information Interfaces and Presentation (e.g. HCI): Miscellaneous

\section{Author Keywords}

cross-device interaction; smartphones

\section{INTRODUCTION}

Decreasing prices and an increasing diversity during the last years led to a noticeable change of the usage and value of computational devices: Smartphones, tablets, smartwatches, or also laptops are our daily companions [7] and shape our leisure behavior as well as our work habits [25]. Accordingly, HCI moved from being concerned with only one artifact (e.g. a laptop) towards addressing an ecology of multiple devices.

The combined use of multiple interactive artifacts substantially increased the complexity of the interactive environment. This led to a new research domain addressing multi-device settings with the goal that individual devices should no longer act like small isolated units but should form an ecology that allows for easy combinations, relations and transfers among them [16].

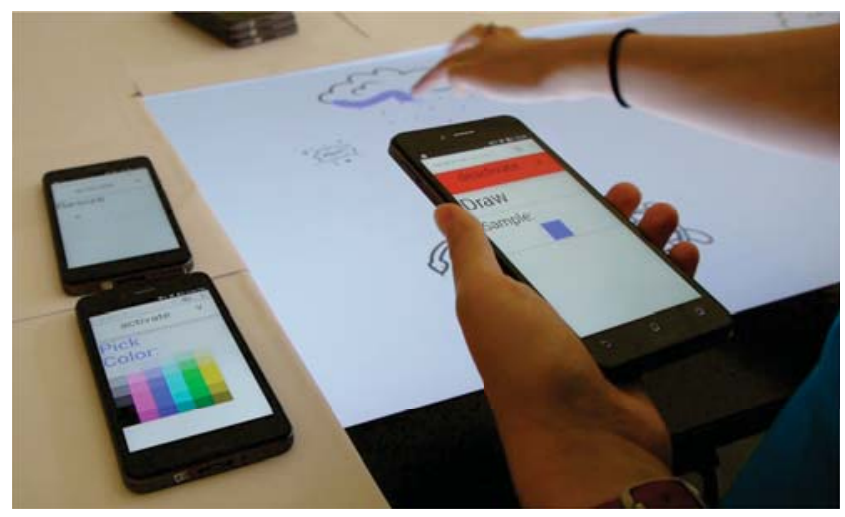

Figure 1. A participant with multiple smartphones in condition phones.

The multitude of available devices encourages the allocation of tasks to specific devices [12] following the concept of traditional tools such as pens or a ruler. However, today's consumer devices are mostly not aware of the existence of other digital companions. Exceptions mainly focus on resource lending or sequential use (e.g. Spotify, Google Chromecast, or Apple's Universal Clipboard) [17]. Generally, the lack of awareness of nearby devices and their incompatibility leads to concerts of notifications, interruptions of workflows due to device and operating system switches, and a potential disuse of resources.

HCI research aims to tackle this problem from a practical as well as a theoretical perspective. On the practical side, research into cross-device interaction has been done to raise mutual awareness of devices, either via additional hardware augmentation (e.g. [22, 23, 28]) or based on software using built-in resources (e.g. [11, 20, 31]). On the theoretical side, working styles known from traditional work practices using pens and rulers have been taken as a basis to establish models that conceptually guide the combination of multiple interactive artifacts into one coherent work environment $[2,19]$. These models propose to design digital functions based on the interaction with physical tools enhanced by computational power resulting in the combination and reuse of functions (e.g. instruments) across different tasks and applications [19].

Most of the named research projects focused on the implementation of systems resembling a working environment consisting of multiple digital and physical artifacts. Research in this area concludes that the distribution of elements in interactive environments is beneficial. However, reviewing existing studies, it is difficult to point out if the influence on users' work behavior is due to the spatial distribution of interface elements or physical affordances of interactive devices. 
To address this, we built a (multi-device) system that allowed us to study how the spatial distribution of tools in an individual work environment is received by participants and to understand the role of physical devices in such a setting.

To observe authentic working behavior, we conducted a user study of an individual creative task that was executed by 20 participants. We studied the effects and influences of spatial distribution of tools on users' interaction with devices, resulting problems and solutions, and task load and user experience. Our findings show that the spatial distribution of interface elements indeed offers advantages, but also elicits new problems that can partly be leveraged by the physical affordances of mobile phones. This paper contributes a summary of benefits and challenges of distributed cross-device desk applications.

\section{RELATED WORK}

While there has been substantial work regarding spatial distribution and physical affordances for distributed interfaces in multi-device collaborative spaces (e.g. [1, 6, 10, 24, 33]), we focus on related work that concentrates on single-user workspaces or facilitates multiple devices as tools/instruments for creative activities. We divide our related work section into two parts: Firstly, we review related work concerned with the digital enhancement of physical desktops resulting in a spatial distribution of artifacts. Secondly, work in the area of cross-device interaction is reviewed that aimed to enhance the computational power of distributed artifacts by using multiple interactive devices.

\section{Augmenting the physical desk}

A first step into an interactive cross-media system was Wellner's DigitalDesk [32]. He projected digital functions onto a physical desk - enabling interaction by using a camera and image processing to track users' actions. Wellner envisioned a system that allows to interact with digital documents in a similar way as one would handle physical sheets of paper.

Gebhardt et al. built upon this approach and developed the Integrative Workplace $[8,9]$, a system that extends physical objects like e.g. printed books with digital functions using an overhead projection and camera system. The Integrative Workplace allows knowledge workers e.g. to excerpt paragraphs or figures out of a printed book, to backtrack them for later reference, and to spatially distribute them on a canvas.

Kidd et al. [18] showed that this spatial distribution is a key factor for successful individual work environments, allowing the user to use space as an additional resource. In our work, we aim to particularly focus on the influence of the spatial distribution of interface elements on users' working behaviour.

\section{From paper to interactive devices}

The concept of spatial distribution has recently been taken further by using digital devices that are combined to enable crossdevice interactions [28, 29]. For example Rädle et al. [28] presented HuddleLamp, a desk lamp with an included camera, that allows for seamless tracking of positions of mobile devices on a desk as well as hands working on them. Results of an evaluation showed that participants are capable of working on multiple surfaces and prefer spatially-aware gestures to manage information transfer and device linkage [29].

Similarly, Hamilton and Wigdor [13] implemented Conductor, a cross-device framework that supports interaction methods that allow for managing several activities across multiple devices in an individual desk environment. Hereby, the focus lied on cross-device settings that allow for easy transition of information as well as for managing relationships between devices in an unobtrusive way. In their study, they show that although participants were not familiar with cross-device settings, they made great use of cross-device functions.

Houben et al. [15] introduced ActivitySpace, a distributed information management system based on the activity theory, which allows users to cope with problems like the lack of transparency or control when working in multi-device environments. The system is based on a configuration space concept, that allows users to manage and distribute applications and resources across available devices. Their study demonstrated how the concept helped users in managing multi-device activities and revealed different usage patterns.

Schmidt et al. [30] explored cross-device interaction techniques for mobile devices and interactive surfaces. For example, the PhoneCopyPaste technique enables users to use phones as physical clipboards and the PhonePalettes technique supports users to move tools from the surface onto mobile devices. With this approach, certain actions are distributed among different devices, and the physical aspect of mobile phones is also being exploited.

Brudy et al. [4] designed CurationSpace, a system based on the theory of instrumental interaction [2] that allows individuals and groups to work with digital artefacts using a shared interactive space and personal smartwatches, that served as a container for multiple instruments (e.g. to change the color or size of an artefact). Their study showed that participants expected the system to work like traditional WIMP-applications (e.g. Microsoft Word) at first. However, they were able to accustom to the new interaction paradigm after some time. We aim to build on Brudy et al.'s [4] work by designing a system incorporating similar instruments/tools for a creative task.

As the related work shows, users are able to cope with a number of multiple devices in order to transfer information and manage different multi-device activities. In particular, users benefit from outsourcing specific functions onto physical devices, successfully exploiting the physical affordances of devices in order to increase the transparency of the workflow. In addition to the spatial distribution of interface elements, we consider the physical affordances of mobile devices the second aspect to be investigated in our study.

\section{RESEARCH QUESTIONS}

The goal of our work is to better understand the benefits and challenges of spatial distribution and physical affordances in an individual work environment. We address this goal by investigating the following research questions:

- RQ1: How do spatial distribution and physical affordances affect users' interaction with devices? 
- RQ2: What kind of problems do participants encounter when working with spatially distributed interface elements and what are their coping mechanisms?

- RQ3: How do the spatial distribution and the physical affordances affect users' task load and user experience?

As we were interested in the comparison of the spatial distribution and the influence of physical devices we implemented three conditions as independent within-subjects variables: (1) a toolbar-based interface (e.g. like Microsoft Paint), (2) an interface with spatially distributed tools on movable panels (e.g. like Adobe Photoshop), and (3) an interface with spatially distributed tools running on mobile phones (like (2) but with physical affordances). We conducted an experimental study of an individual creative activity that was executed by 20 participants. We studied the effect that these three conditions had on the interaction with devices (RQ1), encountered problems and corresponding solutions (RQ2) as well as subjective task load and user experience (RQ3).

\section{USER STUDY}

In order to answer our research questions, we conducted

a counter-balanced within-subjects user study with three different variants of our system as independent variable. We asked participants to perform a creative task with each condition. The dependent variables were interaction behavior (video data analysis and data logs), user task load (NASA TLX), and user experiences (UEQ and semi-structured interview). In the following, we describe in detail our system, the apparatus, the task as well as participants' characteristics, and the procedure of our study.

\section{System}

We built a system based on Webstrates [20] in three different variants: (1) a user interface with a fixed toolbar at the top of a canvas (classic) and two spatially distributed interfaces, (2) one representing tools as digital panels (panels), and (3) one running them on individual smartphones (phones).

The three conditions (see Figure 3) provided the following tools that are known from tool-palettes of applications like Adobe Photoshop (cf. [4, 19]): a brush allowed to draw strokes, a colorpicker could be used to change the color of the brush, a clipboard enabled to copy, paste, cut, or delete objects, a shape drawing tool to draw shapes, a tool to move, and one to resize objects. We decided for six tools to trade-off between providing enough functions to engage in multiple activities and to prevent overwhelming. Previous research has shown that individuals can use up to seven tools [4] and are able to benefit from up to ten tablets in cross-device settings [13].

Each panel/phone had a title and a visual representation of its capabilities (e.g. the color and size of the brush or available colors of the colorpicker). Tools could also be used to change properties of other tools (e.g. colorpicker or resizer). Participants were also able to assign a specific tool to a panel/phone to allow e.g. to have multiple brushes with different colors or sizes. We decided for a simple and basic layout to avoid possible influences of the design and also to allow participants to adapt them according to their preferences.
For panels and phones, tools had to be (de-)activated by pressing a designated (de-)activate-button. On the one hand, this design decision allowed participants to combine multiple tools, on the other hand, this allowed for a better comparability of our three conditions. In the classic condition, participants could select tools as known from traditional toolbar settings by tapping on the corresponding button.

\section{Apparatus}

Figure 3 shows the setting of the study. We chose a 55"tabletop display (Microsoft Perceptive Pixel) with a display resolution of $1920 \times 1080$ pixels as workstation. The actual workspace was limited to an area of the size of $30^{\prime \prime}$ in a 16:9 ratio, a typical size for a personal workspace. This area was the same for all three conditions and was placed in the middle of the screen at the edge where participants were standing during the study. This limited the space where participants could work on their task, as they were not able to move content out of this area. For the classic condition, we placed a toolbar at the top of the workspace. In this condition, the space outside of this area was blank, unused, and no interaction was possible. For the panels condition, we initially placed six different panels representing six tools with the same size of the smartphones used in the phones condition above the workspace, from now on referred to as shelf space. Participants were able to freely move and arrange the panels on the entire shelf space, yet it was not possible to move panels from the shelf space to the workspace. For the phones condition, we placed a cardboard layer on the shelf space. This helped to prevent errors regarding touch recognition when placing the smartphones at the same positions as the panels. As smartphones, we chose six ASUS PadFone Infinity $\left(5^{\prime \prime}, 145 \mathrm{~g}\right)$ with a display resolution of $1920 \times 1080$ pixels, representing a typical size of a smartphone. Participants were able to freely move and arrange the phones on the shelf space in the same way as in the panels condition. We placed a camera in bird's eye-view to record the user study.

\section{Task}

In the study, participants had to perform three individual creative tasks (one for each condition). The task was based on the game 'Story Cubes' ${ }^{1}$. Originally, the game consists of cubes with simplified representations of objects, animals, emotions etc. on each face (see Figure 2). Players have to roll multiple dice at once and use the combination of images to tell a coherent story. Players are free to arrange the dice to support their story. The simplified and icon-like nature of the representations are perceived individually, which allows for varieties of associations and eases getting started.
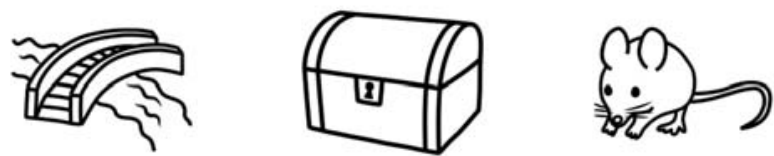

Figure 2. Examples of used images: a bridge (left), a treasure chest (middle), and a mouse (right).

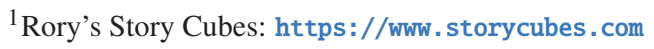



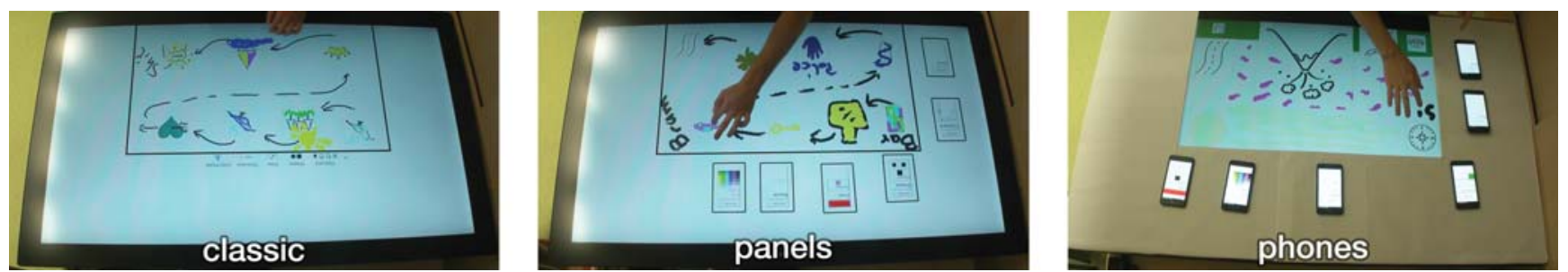

Figure 3. Snapshots of our three different conditions: classic, panels, and phones. Each snapshot was taken during the last third of the specific task.

We adapted the original game play as follows: For each task, we used a randomized subset of 6 out of 82 images (see Figure 2 ), that were not shown before, neither during an initial test phase nor during one of the other conditions. Participants were asked to work with the images and create a short reasonable story based on them. In contrast to the original game play, participants were encouraged to not only move and arrange images, but also add additional content (e.g. draw objects, change content, resize or multiply images) using the available tools.

The adaption of the original game play enabled participants to extend the creative potential towards visual sequences, combinations and extensions of given images, or painting-like results. Yet, they were free in the choice of tools and not forced to make use of them.

We designed the task to encourage a heavy use of the different provided tools to see how participants deal with them in the different conditions. This allowed us to provide a nuanced description of tool usage depending on their spatial distribution and their physical affordances.

\section{Participants}

20 participants ( 11 female, 9 male) were recruited for the study. The mean age was 25.2 years $(\mathrm{SD}=4.2$, aged 20 39). Two participants were left-handed. All participants had normal or corrected to normal vision - consequently they had no problems with the visual perception of the different interfaces. To recruit participants we used flyers looking for creative people that are interested in interactive environments.

\section{Procedure}

At the beginning of each study, participants were asked to fill out a questionnaire about demographics. Then, they were introduced to their task in one of the conditions (classic, panels, or phones) with a sample image set. Participants received an introduction into all condition-specific features and were given an adequate amount of time to explore its functionality with sample images until they felt comfortable enough using it. The experimenter then started the task giving participants a time limit of ten minutes. During each task, general questions about the procedure or the system were answered but they were not supported in their task. Then, participants were asked to fill out a NASA TLX [14] and a User Experience Questionnaire (UEQ) [21]. This sequence was the same for all three conditions. We counter-balanced the order of the three conditions to minimize carry-over effects. After the last task, we conducted a short semi-structured interview to ask participants about their strategy, their activities, and their preferences. Each session lasted about 90 minutes, and afterwards participants were compensated for their time.

\section{DATA ANALYSIS}

We used data logging to quantify participants' interactions with the systems. In addition, we coded videos regarding breakdowns [3] to analyze participants' encountered problems and solutions to overcome them. In order to investigate participants' task load and user experience, we employed two questionnaires and a concluding interview.

\section{Data Logs}

We logged the number and durations of participants' use of tools. This allowed us to investigate participants' interactions and analyze the effect of spatial distribution and physical affordances on participants' interaction behavior (see RQ1).

\section{Video Data}

We developed a coding scheme to analyze our video data focusing on breakdowns [3]. According to Bødker [3], "[b]reakdowns related to the use process occur when work is interrupted by something [...]. [B]reakdowns [are] somehow caused by the computer application."

We focused on participants' breakdowns when interacting with the conditions, as well as their employed working strategies and behavior to overcome them. After an iterative analysis of a subset of the video data, we distinguished between two categories of breakdowns: tool and object.

Tool breakdowns are described as problems occurring with the application of a tool (e.g. problems with activating/selecting a tool). Object breakdowns are problems concerning the definition of what constitutes an object (e.g. problems with the combination of several objects into one). Inter-coder reliability for our video coding was tested with a sample of ten minutes for each condition by our two independent coders. Cohen's Kappa with a value of $\varkappa=0.74$ revealed a substantial intercoder reliability. We used the video data for the quantitative as well as qualitative analysis of breakdowns (see RQ2).

\section{Questionnaires and Interview}

After each of the three conditions, participants were asked to fill out a NASA TLX questionnaire to understand the task load employed for the specific condition, and the UEQ, focusing on user experience aspects. We concluded each session with a semi-structured interview asking questions regarding strategies, preferences, and general comments (see RQ3). 


\section{FINDINGS \& DISCUSSION}

The following subsections are structured according to our research questions. The combination of data logging, video analysis, questionnaires, and a semi-structured interview allows for data-triangulation as well as a quantitative and qualitative analysis and discussion of the data. We analyzed the data using a one-way repeated measure ANOVA with pairwise post-hoc comparisons (including Bonferroni correction).

\section{RQ1: Influences on Interaction}

In this section, we distinguish between tool use and arrangement of the workspace.

\section{Tool Use}

Table 1 reports the number of times participants used a specific tool across the three conditions. The analysis revealed statistically significant differences for using the brush $(F(2,38)=22.663, p<.05)$. Pairwise post-hoc comparisons showed statistically significant differences between classic and panels $(p<.016)$ as well as classic and phones $(p<.016)$. There was also a statistically significant difference for using the clipboard $(F(2,38)=4.876, p<.05)$. However, pairwise post-hoc comparisons showed no statistically significant differences after applying Bonferroni correction. There were no statistically significant differences for the other tools.

\begin{tabular}{l|lll} 
& classic & panels & phones \\
\hline Brush* & $19.33^{\mathrm{a}, \mathrm{b}}(6.35)$ & $10.32^{\mathrm{a}}(5.59)$ & $11.94^{\mathrm{b}}(6.30)$ \\
Clipboard* & $5.23(4.32)$ & $2.77(2.18)$ & $2.94(2.58)$ \\
ColorPicker & $2.71(1.39)$ & $3.48(1.88)$ & $2.88(1.01)$ \\
Move & $9.49(2.83)$ & $10.99(5.03)$ & $9.38(5.11)$ \\
Resize & $2.23(2.38)$ & $2.31(1.52)$ & $1.95(1.29)$ \\
Shapes & $0.68(0.79)$ & $0.88(1.09)$ & $0.63(0.66)$
\end{tabular}

Table 1. Mean frequency of tool uses. Statistically significant differences are marked by asterisks, raised letters show statistically significant differences for post-hoc tests. Standard deviation (SD) is shown in brackets.

These results are also reflected in our qualitative observations of the video data. In classic, participants often used the images and embedded them into painting-like arrangements, including additionally drawn image-like elements on the canvas (see Figure 4). In the interview, some mentioned that they could use their pre-existing knowledge (P4: "I know how it works.", P16: "I am familiar with it and didn't have to think.", P15: "It was intuitive because I already know similar things."). Reflecting on their interaction strategy, P13 said "In [classic] I moved and painted alternating.", and P20 mentioned "I drew more in [classic]." In classic, participants often altered or discarded parts of their drawings, resulting in a heavier usage of the clipboard.

In conditions panels and phones, participants worked differently. The reduced use of the brush was especially observable when focusing on the objects they were drawing on. In classic, participants drew equally on given images and the canvas. In contrast to that, with panels and phones, participants drew mostly on images and used the canvas for connecting arrows, annotations or similar (see Figure 5). Also, they chose to combine and nest multiple images, instead of drawing. One participant e.g. combined images to create a starry sky in

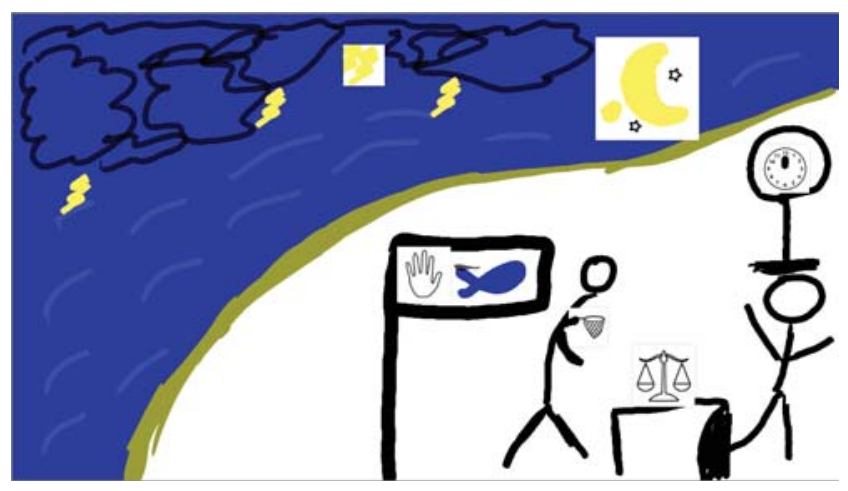

Figure 4. Typical for classic: P12 focused on drawing on the canvas.

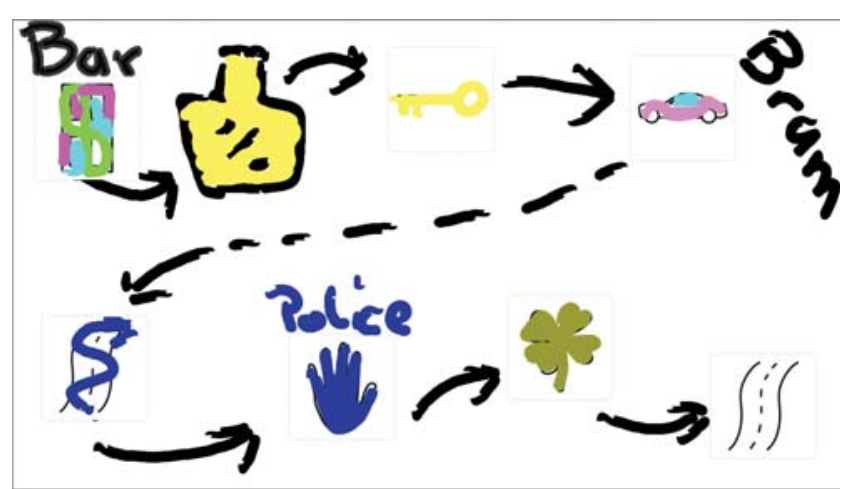

Figure 5. Typical for panels and phones: P11 enhanced given images and used the canvas for annotations.

phones. The same participant chose to draw water instead of combining multiple images to create a lake in classic.

When asking participants how panels and phones influenced them, they stated that "I first arranged all images and then I started drawing. [This helped] to structure thoughts."(P13) P1 stated that "it is more efficient to turn [a tool] on and do it to all of the images, instead of switching modes any time like in [classic]." P9 said "I didn't want to choose the picture and then see what I do with it, it was better to choose a tool and see what I could do with it." However, participants thought it was "confusing to have separate devices" (P10) and "artificial to have so many phones" (P15). P11 mentioned that "having less than six phones could have been better."

Summarizing, we observed differences in the use of tools on a quantitative level, especially for the brush and clipboard, resulting in more complex approaches to work with provided images for panels and phones. Participants adapted their working styles to consecutive usages of a single tool to increase efficiency, which was supported by a higher flexibility concerning the arrangement of their workspace. Yet, participants hesitated to exploit the potentials of physical phones, which was observable in the minimal physical movements of devices and by the unused possibility to have multiple representations of a tool. 


\section{Arrangement of the Workspace}

Conditions panels and phones allowed participants to move tools and by this arrange the workspace according to their needs. We expected a heavy use of this possibility, especially for phones due to their physical affordance. We noted the times participants moved their tools: Seven participants moved panels and nine participants moved phones - only two of them moved tools in both conditions. Interestingly, this arrangement of the workspace happened mainly in the beginning of a task, before participants started working with the images. Only two participants moved the tools during the actual task.

When asked about the benefits, participants answered "...it's a phone, I know I can move a phone around." (P1), "I moved all phones to one side to be able to see them in one glance." (P3), "It was good to set up tools in space." (P6), and "I moved all phones to the right because I am right handed." (P9). When asked about what discouraged them to move tools, they said "I was scared to move the phones because maybe they are connected in some way and it would break when moving." (P5), or "I was too concentrated in thinking about the story." (P4)

The unfamiliar experience of using multiple physical devices and the resulting hesitation regarding their physical arrangement can be interpreted as a "legacy bias" [26, 27]. Users are influenced by pre-existing knowledge about common WIMPinterfaces and technologies used e.g. in laptops. Although participants received an introduction into all features of each condition and were given an adequate amount of time to try it on their own before the actual task started, they only reluctantly made use of the benefits of physical devices (e.g. move them to arrange an individual and task-specific layout).

\section{Influences on Interaction}

\section{Benefits}

- Spatial Distribution of panels and phones changes working approaches, which leads to a subjective estimation of more efficiency and flexibility.

\section{Challenges}

- Participants hesitated to exploit the potentials of physical devices (phones) but arranged their workspaces before starting the actual task.

\section{RQ2: Encountered Problems and Coping Mechanisms}

In order to address RQ2, we distinguish between tool and object breakdowns. Table 2 shows the mean number of breakdowns across the three conditions. In the subsequent sections, we discuss reasons and implications of the two kinds of breakdowns based on our qualitative analysis. Finally, we dig deeper into participants' learning behaviour, in particular the way they worked with and adapted to panels and phones, described from a qualitative point of view.

\begin{tabular}{l|lll} 
& classic & panels & phones \\
\hline Tool* $^{*}$ & $4.45^{\mathrm{a}}(3.55)$ & $9.70^{\mathrm{a}}(6.94)$ & $6.20(4.11)$ \\
Object & $1.55(1.73)$ & $1.50(1.61)$ & $2.25(1.89)$
\end{tabular}

Table 2. Mean number of breakdowns. Statistically significant differences are marked by asterisks, raised letters show statistically significant differences for post-hoc tests. SD is shown in brackets.

\section{Analysis of Tool Breakdowns}

Table 2 shows the mean number of breakdowns per condition. The analysis of tool breakdowns revealed a statistically significant difference in the frequency of tool breakdowns across the three conditions $(F(2,38)=6.380, p<.05)$. Pairwise post-hoc comparisons showed a statistically significant difference when comparing classic and panels $(p<.016)$. However, no other post-hoc comparison showed statistically significant differences. The fact that only panels resulted in a significantly higher number of tool breakdowns than classic suggests that the physical affordances of phones helped participants to cope with the spatial distribution, resulting explicitly in less tool breakdowns.

The clear spatial and semantic separation of workspace and toolbar in classic caused less breakdowns. The predefined layout was perceived to be "more organized." (P4) and participants "didn't have to look away." (P17) P5 characterized classic as "it all belongs together." In condition classic, it was clear to participants, that a tool had to be activated prior to its usage and that a selected tool could be used to modify objects. Furthermore, this condition was closest to common WIMP-applications and participants activated and deactivated functions easily. In cases when they had problems (e.g. a participant wants to move an object but the brush is still activated), they would quickly understand why the problem occurred and were able to reverse it easily without interruption in their workflow. Compared to conditions panels and phones, we observed fewer breakdowns and less problems in total.

In contrast to that, participants working with conditions panels and phones had more problems with the activation and deactivation of tools. The video data showed that participants especially working with panels often activated a tool while another one was still activated - resulting in unintentional effects like drawing instead of moving (e.g. activating move while brush is still activated results in drawing a stroke and moving an object). P5 said "I didn't understand in the beginning how activating and deactivating works." and P19 described "leaving a tool activated and drawing by mistake was annoying." The activation of tools especially in panels led to more breakdowns due to two reasons: (1) switching the state of a tool involved treating a tool as an object and using the activation button as a meta-tool and (2) the flexibility of moving tools and the basic layout of them might have caused difficulties to find specific tools. In addition, it was also unclear to some participants what tool(s) had to be active in order to successfully perform an activity (P9 mentioned: "I thought that to change the color I also had to activate the brush.")

Also, we observed that participants were reluctant to apply a tool to another one (e.g. to apply the colorpicker to the brush to change its color). They were in the mindset that one tool 
mapped one functionality and had problems understanding that more than one tool could be activated. Applying one tool onto another one required participants treating one tool as an object and - especially in the beginning - they had trouble with that. They suggested to customize specific tools to leverage this confusion (P14 said "changing the color of some tools could be useful to know which is most important.")

Our findings show that the tool breakdowns in panels and phones forced participants to focus on how the tools work, which helped them to break out of their common way of usage and adapt to the spatially distributed interface. This was particularly supported by physical devices (phones). Focusing more on tools, several participants mentioned that they switched their focus from performing operations on a selected image to performing a certain operation on all objects (P13: "...[it was possible to separate] the activities more... first move and then resize, instead of doing it all at once... and this ordered ones thoughts more.")

\section{Analysis of Object Breakdowns}

The analysis of object breakdowns revealed no statistically significant differences across the conditions (see Table 2).

Looking at the results that participants created throughout the sessions, we could observe differences between classic and panels \& phones. Participants used the brush more often in classic, which led to the fact that they created many new objects on the canvas throughout the session (e.g. P20 mentioned "...it was much quicker and easier, so that's why I drew more.") Although they also resized and moved the provided images, , they did not draw on them extensively, which led to a clear distinction between created objects and provided images. We observed, that in classic, participants understood quite easily how to create new objects from the beginning on. As they had only few problems, they were not required to learn or change their behavior throughout the session, which led to a quite stable and consistent way of working.

In contrast to that, participants took on a more incremental way of working with objects in panels and phones. Here, they focused more on refining and extending the provided images using the brush and shapes. Also, they combined and nested multiple images. While they focused on these images, there were two main subtypes of object breakdowns:

(1) Object-differentiation breakdowns: Participants combined multiple images (e.g. placing an airplane in a field of stars) and tried to move this new object. The system did not recognize the combination of images as one object, which resulted in the movement of single parts. Similar breakdowns happened when participants drew an additional figure consisting of multiple strokes. Moving this object-to-be resulted in moving single strokes.

(2) Consistent-behavior-breakdowns: In these two conditions participants often tried to fill zoned areas of images with a selected color (e.g. the river underneath the bridge in Figure 2 left). As this worked perfectly for the entire canvas or self-drawn strokes, it was not possible for zoned areas of images. Participants expected that tools work consistently on all objects.

\section{Analysis of Learning Behaviour}

We observed that the flexible use and combination of tools as well as the seamless switch between tools and objects led to more problems but also to a more advanced way of applying and combining tools in panels and phones. Participants were frequently required to consciously try and understand the tools and the system as a whole in panels and phones compared to classic. Whereas in classic, participants were able to reverse problems easily without taking off the focus on their task, participants in panels and phones were regularly required to consciously focus on the functionality and usage of the tools which distracted them from their actual task. However, this conscious focus on the system led to the fact that they increasingly understood the system throughout the session and became bolder in trying out new combinations of tools and objects.

We could also observe that participants working in panels and phones adapted to the condition throughout the session as they got used to (de-)activating tools and had fewer problems applying other tools on them and thus treating them like objects. This was especially the case for five participants who discovered possible tool-tool interactions beyond the ones explained and used these to their advantage (e.g. P7 colored the background of a panel to support recognition in panels).

Overall, we observed that participants in conditions panels and phones took on a slightly different strategy during the session. As they had more problems understanding the functionality and structure of the different tools, they would start quite slowly with moving objects around. When drawing, they would start with hesitantly trying some strokes. We observed that participants already had some problems at this level (e.g. the colorpicker needs to be activated to color the brush, but then needs to be deactivated before drawing, otherwise the color of the background will change). However, we also observed that these breakdowns not only had a negative effect on the participants as they caused problems but also led participants to learn about the structure of the system and to try out new things as they progressed throughout the session. In contrast to condition classic, participants in panels and phones would then proceed to not mainly draw on the canvas but to try and combine different objects. For example, they often drew on given objects, or deliberately copied and inserted objects into already existing ones. This would in turn lead to an advanced set of new problems.

For example, participants increasingly combined existing objects into more complex objects and would then switch between applying tools on objects and/or parts of it. This would then bring up the question of what constitutes an object and how to distinguish between different levels of objects. Again, participants would experience many breakdowns concerning this issue but would eventually learn about it. Thus, we conclude that the more flexible settings in panels and phones led to more problems, but also led to a steeper learning curve and encouraged more advanced/powerful interaction, particularly concerning the combination of different tools and objects. 


\section{Encountered Problems and Coping Mechanisms}

\section{Benefits}

- Using tools on physical devices (phones) does not result in statistically significant more breakdowns than using a toolbar setting (classic).

- Solving problems in panels and phones guides participants to actively engage with the system, learn, and adapt their approach.

- Participants learn to cope with breakdowns in panels and phones over time.

- Participants combine tools and nest objects in flexible and creative ways.

\section{Challenges}

- Participants had difficulties with the awareness of the properties of the tools and with using multiple tools in combination in panels and phones.

- The (de-)activation of tools in panels and phones caused troubles.

- Participants had troubles with an inconsistent behavior of using a tool on different objects.

\section{RQ3: Task Load and User Experience}

This section reports our results regarding task load and user experience.

\section{Task Load}

Table 3 reports the mean values of participants' subjective ratings of the NASA TLX scores for all three settings. The analysis revealed a statistically significant difference for the overall task load $(F(2,38)=4.774, p<.05)$, however pairwise post-hoc comparisons showed no statistically significant differences after applying Bonferroni correction.

\begin{tabular}{l|lll} 
& classic & panels & phones \\
\hline Total* & $28.63(21.99)$ & $37.79(13.68)$ & $37.66(16.67)$ \\
Mental Demand* & $34.50(21.99)$ & $45.00(22.18)$ & $48.50(22.13)$ \\
Physical Demand* & $25.50(17.91)$ & $40.50(24.06)$ & $32.50(20.29)$ \\
Performance* & $20.79(21.10)$ & $33.50(21.53)$ & $39.65(29.40)$ \\
Effort* & $32.00^{\mathrm{a}}(19.70)$ & $44.25^{\mathrm{a}}(20.73)$ & $37.50(21.67)$
\end{tabular}

Table 3. Mean values of overall task load and subscales with statistically significant differences - marked by asterisks and raised letters (posthoc). SD is shown in brackets.

Analysis of mean values of the TLX subscales showed statistically significant differences regarding mental demand $(F(2,38)=4.372, p<.05)$, physical demand $(F(2,38)=4.469$, $p<.05)$, the estimation of their performance $(F(2,38)=6.390$, $p<.05)$, and effort $(F(2,38)=4.786, p<.05)$. None of the pairwise comparisons showed statistically significant differences except for the subscale effort, which showed a statistically significant difference between classic and panels $(p<.016)$.
Although the mean values suggest that the classic condition resulted in lower task load than the panels and phones conditions, this difference was not found to be statistically significant in pairwise comparisons except for effort. As the conditions panels and phones introduced an unfamiliar interaction environment, we expected a much larger difference in task load scores as participants had to adapt to a new way of handling tools and applying them to objects in these conditions.

Regarding the subscale effort, results show that participants working in condition panels rated the effort that the use of the system required statistically significant higher than condition classic. There were - however - no statistically significant differences between condition classic and phones. This suggests that distributing tools from a toolbar to panels leads to more effortful interaction, however, this increased effort can be mediated by physical affordances of phones. P16 described that "it's easier to manipulate physical objects, manipulating digital objects is harder", and continued "[phones] gives a more direct feeling, because of the physical objects."

\section{User Experience}

Table 4 shows the mean scores of the User Experience Questionnaire for the three conditions. The analysis revealed statistically significant differences for the subscales attractiveness $(F(2,38)=3.765, p<.05)$, perspicuity $(F(2,38)=5.261$, $p<.05)$, and efficiency $(F(2,38)=5.888, p<.05)$. Pairwise post-hoc comparisons showed no statistically significant differences after applying Bonferroni correction. In addition, the subscales dependability, stimulation, and novelty did not show statistically significant differences.

\begin{tabular}{l|lll} 
& classic & panels & phones \\
\hline Attractiveness* & $1.43(0.96)$ & $0.89(0.86)$ & $0.82(1.32)$ \\
Perspicuity* & $1.94(0.98)$ & $1.10(0.90)$ & $1.15(1.17)$ \\
Efficiency* & $1.16(0.99)$ & $0.36(1.04)$ & $0.46(1.28)$
\end{tabular}

Table 4. Aspects of user experience with statistically significant differences - marked by asterisks. SD is shown in brackets. Min: -3 / Max: 3 - the higher the better.

The mean values suggest higher scores and thus a more positive user experience for classic compared to panels and phones, but these differences were not found to be statistically significant different. Although we introduced a novel and unfamiliar interaction environment, the user experiences did not change dramatically. In general, all subscales showed positive mean values, indicating a good user experience for all conditions. The subscale perspicuity, which focuses on the understandability showed high values over all conditions, which means that participants were able to understand how to interact with the tools in the different conditions equally. This was further verified by the fact that there were no statistically significant differences for the subscales dependability, stimulation, and novelty.

Interestingly, participants mentioned that both, panels and phones helped them to change their workflow and be more efficient (see RQ1: Influences on Interaction), yet there was no statistically significant difference for the subscale efficiency for pairwise comparisons. 
Task Load and User Experience

\section{Benefit}

- Panels and phones result in similar task load and user experiences compared to classic.

\section{Challenge}

- Participants report a higher effort when working with spatially distributed panels.

\section{IMPLICATIONS FOR RESEARCH AND DESIGN}

Our experimental study showed several benefits of Spatial Distribution of tools and Physical Affordances. That resulted in an adaption of participants' workflows: There was a change from alternating different tools and editing single objects with them towards a tool-focused approach, where participants used one tool and applied it to several objects, which was considered to be more efficient and helped to structure thoughts. Also, the possibility to arrange the workspace by moving tools was mentioned to be helpful. Participants further benefit from the physical affordances of phones as they leveraged the statistically significant difference in the number of breakdowns between panels and classic. Task load and user experiences across the three conditions were also comparable.

One of the challenges we identified was that participants hesitated to exploit the full potentials of the physical affordances of phones due to a possible "legacy bias" [26, 27]. This seems to be a general problem in the field of cross-device interaction: Users are often influenced by their pre-existing knowledge and established interaction with WIMP-applications on their personal computers. This leads to comparing tablets with application windows [13], using single tablets instead of exploiting full potentials of multi-tablet systems [5, 27], or as in our study the hesitation to make full use of the physical affordances of phones.

One of the main challenges was the activation of tools and the resulting lack of awareness of tools states. In our experiment, we decided for an explicit activation using a designated button to have comparable actions across our three conditions. Although this required participants to focus more on the specific tools, they often forgot to (de-)activate tools, resulting in troubles. In a cross-device setting using physical tools, a more implicit way of activation could be useful: Tools could be activated by simply holding them, making use of built-in sensors. The result would be a clear state of tool activation and an analogy to real world tools (compare the tool colorpicker with a painter's sample board). Although this would be useful for single tools, it might lead to new challenges regarding the combination of multiple tools or assembling of tools.

Participants expected a consistent behavior of tools across different types of objects. In our case, they expected to be able to fill all types of objects (canvas, images, and self-drawn elements) using the colorpicker. This means that users would expect to be able to make use of a tool like a brush across several applications, resulting in the same behavior: drawing a stroke. A similar example is presented in [19]: Here the authors refer to the analogy of a painter, who can use his brushes to paint on a canvas or on a wall. They use this example to provide their interpretation of instruments in (ubiquitous) instrumental interaction - instruments should be usable on different surfaces when it makes sense even if this interaction was not intended by a designer of the specific system [19]. To allow for the proper implementation of a multi-device work environment completely based on the interaction paradigm instrumental interaction, Klokmose and Beaudouin-Lafon [19] present a software architecture that was explicitly developed for guiding the development of instruments. It would be interesting to see if future systems that are explicitly based on instrumental interaction could potentially address the identified challenges and extend the named benefits of our study.

\section{LIMITATIONS}

There are two main aspects of our study that limit the generalizability of our findings. First, our task with 6 randomized images per condition might have influenced our participants: The simplified and monochrome representations might have influenced participants in the way they used tools like the brush or the colorpicker. Future work could investigate to what extent our findings are also visible in settings beyond creative activities (e.g. sensemaking). Secondly, with conditions panels and phones we introduced a novel interaction environment, which differs from traditional WIMP interfaces as interface elements are not only physically separated from the workspace (phones) but it was also possible to alter tools using other tools (panels and phones). This introduction caused a change of thinking for participants. The given time of ten minutes might have been too short to adapt to this new interaction environment completely. In our study, we leveraged this problem by giving participants an adequate amount of time to get used to the system and practice interaction in each of the three conditions.

\section{CONCLUSION}

In this paper, we reported on findings from an experimental user study of an individual creative task that was executed by 20 participants. We studied the effects and influences of spatially distributed tools and physical affordances on users' interaction with devices, resulting problems and solutions, and task load and user experience. Our results reveal obstacles that participants encountered when working with spatially distributed tools. However, we could also show that the affordances of physical devices can leverage some of these problems. In order to reap the benefits of spatial distribution and physical affordances and address elicited challenges, we suggest that future research should draw on the concept of instrumental interaction.

\section{ACKNOWLEDGMENTS}

We thank the German Research Foundation (DFG) for financial support within project C01 of SFB/Transregio 161.

\section{REFERENCES}

1. Jakob Bardram, Sofiane Gueddana, Steven Houben, and Søren Nielsen. 2012. ReticularSpaces: Activity-based Computing Support for Physically Distributed and 
Collaborative Smart Spaces. In Proceedings of the SIGCHI Conference on Human Factors in Computing Systems (CHI'12). ACM, New York, NY, USA, 2845-2854. DOI :

http://dx.doi.org/10.1145/2207676.2208689

2. Michel Beaudouin-Lafon. 2000. Instrumental Interaction: An Interaction Model for Designing post-WIMP User Interfaces. In Proceedings of the SIGCHI Conference on Human Factors in Computing Systems (CHI '00). ACM, New York, NY, USA, 446-453. DOI : http://dx.doi.org/10.1145/332040.332473

3. Susanne Bødker. 1995. Context and Consciousness. Massachusetts Institute of Technology, Cambridge, MA, USA, Chapter Applying Activity Theory to Video Analysis: How to Make Sense of Video Data in Human-computer Interaction, 147-174. http: //dl . acm.org/citation. cfm?id=223826.223833

4. Frederik Brudy, Steven Houben, Nicolai Marquardt, and Yvonne Rogers. 2016. CurationSpace: Cross-Device Content Curation Using Instrumental Interaction. In Proceedings of the 2016 ACM on Interactive Surfaces and Spaces (ISS '16). ACM, New York, NY, USA, 159-168. DOI : http://dx.doi.org/10.1145/2992154.2992175

5. Nicholas Chen, François Guimbretière, and Abigail Sellen. 2013. Graduate Student Use of a Multi-slate Reading System. In Proceedings of the SIGCHI Conference on Human Factors in Computing Systems (CHI '13). ACM, New York, NY, USA, 1799-1808. DOI : http://dx.doi.org/10.1145/2470654.2466237

6. Haeyong Chung, Chris North, Jessica Zeitz Self, Sharon Chu, and Francis Quek. 2014. VisPorter: facilitating information sharing for collaborative sensemaking on multiple displays. Personal and Ubiquitous Computing 18, 5 (01 Jun 2014), 1169-1186. DOI : http://dx.doi .org/10.1007/s00779-013-0727-2

7. Linda Di Geronimo, Maria Husmann, and Moira C. Norrie. 2016. Surveying Personal Device Ecosystems with Cross-device Applications in Mind. In Proceedings of the 5th ACM International Symposium on Pervasive Displays (PerDis '16). ACM, New York, NY, USA, 220-227. DOI : http://dx.doi.org/10.1145/2914920.2915028

8. Christoph Gebhardt, Roman Rädle, and Harald Reiterer. 2014a. Employing Blended Interaction to Blend the Qualities of Digital and Physical Books. In Mensch \& Computer 2014 - Tagungsband, Andreas Butz, Michael Koch, and Johann Schlichter (Eds.). De Gruyter Oldenbourg, Berlin, 35-44. DOI :

http://dx.doi .org/10.0.5.235/icom. 2014.0028

9. Christoph Gebhardt, Roman Rädle, and Harald Reiterer. 2014b. Integrative Workplace: Studying the Effect of Digital Desks on Users' Working Practices. In CHI '14 Extended Abstracts on Human Factors in Computing Systems (CHI EA '14). ACM, New York, NY, USA, 2155-2160. DOI :

http://dx.doi .org/10.1145/2559206.2581186
10. Tony Gjerlufsen, Clemens Nylandsted Klokmose, James Eagan, Clément Pillias, and Michel Beaudouin-Lafon. 2011. Shared Substance: Developing Flexible Multi-surface Applications. In Proceedings of the SIGCHI Conference on Human Factors in Computing Systems (CHI '11). ACM, New York, NY, USA, 3383-3392. DOI : http://dx.doi.org/10.1145/1978942.1979446

11. Jens Grubert and Matthias Kranz. 2017. HeadPhones: Ad Hoc Mobile Multi-Display Environments Through Head Tracking. In Proceedings of the 2017 CHI Conference on Human Factors in Computing Systems (CHI '17). ACM, New York, NY, USA, 3966-3971. DOI : http://dx.doi.org/10.1145/3025453.3025533

12. Jonathan Grudin. 2001. Partitioning Digital Worlds: Focal and Peripheral Awareness in Multiple Monitor Use. In Proceedings of the SIGCHI Conference on Human Factors in Computing Systems (CHI '01). ACM, New York, NY, USA, 458-465. DOI : http://dx. doi.org/10.1145/365024.365312

13. Peter Hamilton and Daniel J. Wigdor. 2014. Conductor: Enabling and Understanding Cross-device Interaction. In Proceedings of the SIGCHI Conference on Human Factors in Computing Systems (CHI '14). ACM, New York, NY, USA, 2773-2782. DOI : http://dx.doi.org/10.1145/2556288.2557170

14. Sandra G Hart and Lowell E Staveland. 1988. Development of NASA-TLX (Task Load Index): Results of empirical and theoretical research. Human mental workload 1, 3 (1988), 139-183. DOI : http://dx.doi.org/10.1016/S0166-4115(08)62386-9

15. Steven Houben, Paolo Tell, and Jakob E. Bardram. 2014. ActivitySpace: Managing Device Ecologies in an Activity-Centric Configuration Space. In Proceedings of the Ninth ACM International Conference on Interactive Tabletops and Surfaces (ITS '14). ACM, New York, NY, USA, 119-128. DOI :

http://dx. doi .org/10.1145/2669485.2669493

16. Hans-Christian Jetter, Harald Reiterer, and Florian Geyer. 2014. Blended Interaction: understanding natural human-computer interaction in post-WIMP interactive spaces. Personal and Ubiquitous Computing 18, 5 (01 Jun 2014). DOI : http://dx. doi.org/10.1007/s00779-013-0725-4

17. Tero Jokela, Jarno Ojala, and Thomas Olsson. 2015. A Diary Study on Combining Multiple Information Devices in Everyday Activities and Tasks. In Proceedings of the 33 rd Annual ACM Conference on Human Factors in Computing Systems (CHI'15). ACM, New York, NY, USA, 3903-3912. DOI :

http://dx.doi.org/10.1145/2702123.2702211

18. Alison Kidd. 1994. The Marks Are on the Knowledge Worker. In Proceedings of the SIGCHI Conference on Human Factors in Computing Systems (CHI '94). ACM, New York, NY, USA, 186-191. DOI : http://dx.doi.org/10.1145/191666.191740 
19. Clemens Nylandsted Klokmose and Michel Beaudouin-Lafon. 2009. VIGO: Instrumental Interaction in Multi-surface Environments. In Proceedings of the SIGCHI Conference on Human Factors in Computing Systems (CHI '09). ACM, New York, NY, USA, 869-878. DOI : http://dx.doi.org/10.1145/1518701.1518833

20. Clemens Nylandsted Klokmose, James R. Eagan, Siemen Baader, Wendy Mackay, and Michel Beaudouin-Lafon. 2015. Webstrates: Shareable Dynamic Media. In Proceedings of the 28th Annual ACM Symposium on User Interface Software \& Technology (UIST '15). ACM, New York, NY, USA, 280-290. DOI :

http://dx.doi.org/10.1145/2807442 .2807446

21. Bettina Laugwitz, Theo Held, and Martin Schrepp. 2008. Construction and Evaluation of a User Experience Questionnaire. In Proceedings of the 4th Symposium of the Workgroup Human-Computer Interaction and Usability Engineering of the Austrian Computer Society on HCI and Usability for Education and Work (USAB '08). 63-76. DOI : http://dx.doi.org/10.1007/978-3-540-89350-9_6

22. Andrés Lucero, Jussi Holopainen, and Tero Jokela. 2011. Pass-them-around: Collaborative Use of Mobile Phones for Photo Sharing. In Proceedings of the SIGCHI Conference on Human Factors in Computing Systems (CHI '11). ACM, New York, NY, USA, 1787-1796. DOI : http://dx.doi .org/10.1145/1978942 .1979201

23. Nicolai Marquardt, Ken Hinckley, and Saul Greenberg. 2012. Cross-device Interaction via Micro-mobility and F-formations. In Proceedings of the 25th Annual ACM Symposium on User Interface Software and Technology (UIST '12). ACM, New York, NY, USA, 13-22. DOI : http://dx . doi . org/10.1145/2380116.2380121

24. Roberto Martinez-Maldonado, Peter Goodyear, Judy Kay, Kate Thompson, and Lucila Carvalho. 2016. An Actionable Approach to Understand Group Experience in Complex, Multi-surface Spaces. In Proceedings of the 2016 CHI Conference on Human Factors in Computing Systems (CHI '16). ACM, New York, NY, USA, 2062-2074. DOI : http://dx.doi.org/10.1145/2858036.2858213

25. Fabrice Matulic, Maria Husmann, Seraiah Walter, and Moira C. Norrie. 2015. Eyes-Free Touch Command Support for Pen-Based Digital Whiteboards via Handheld Devices. In Proceedings of the 2015 International Conference on Interactive Tabletops \& Surfaces (ITS '15). ACM, New York, NY, USA, 141-150. DOI : http://dx . doi . org/10.1145/2817721.2817728

26. Meredith Ringel Morris, Andreea Danielescu, Steven Drucker, Danyel Fisher, Bongshin Lee, m. c. schraefel, and Jacob O. Wobbrock. 2014. Reducing Legacy Bias in Gesture Elicitation Studies. interactions 21, 3 (May 2014), 40-45. DOI : http://dx. doi .org/10.1145/2591689
27. Thomas Plank, Hans-Christian Jetter, Roman Rädle, Clemens N. Klokmose, Thomas Luger, and Harald Reiterer. 2017. Is Two Enough?! Studying Benefits, Barriers, and Biases of Multi-Tablet Use for Collaborative Visualization. In Proceedings of the 2017 CHI Conference on Human Factors in Computing Systems (CHI '17). ACM, New York, NY, USA, 4548-4560. DOI : http://dx.doi.org/10.1145/3025453.3025537

28. Roman Rädle, Hans-Christian Jetter, Nicolai Marquardt, Harald Reiterer, and Yvonne Rogers. 2014. HuddleLamp: Spatially-Aware Mobile Displays for Ad-hoc Around-the-Table Collaboration. In Proceedings of the Ninth ACM International Conference on Interactive Tabletops and Surfaces (ITS '14). ACM, New York, NY, USA, 45-54. DOI :

http://dx.doi.org/10.1145/2669485.2669500

29. Roman Rädle, Hans-Christian Jetter, Mario Schreiner, Zhihao Lu, Harald Reiterer, and Yvonne Rogers. 2015. Spatially-aware or Spatially-agnostic?: Elicitation and Evaluation of User-Defined Cross-Device Interactions. In Proceedings of the 33rd Annual ACM Conference on Human Factors in Computing Systems (CHI'15). ACM, New York, NY, USA, 3913-3922. DOI : http://dx.doi.org/10.1145/2702123.2702287

30. Dominik Schmidt, Julian Seifert, Enrico Rukzio, and Hans Gellersen. 2012. A Cross-device Interaction Style for Mobiles and Surfaces. In Proceedings of the Designing Interactive Systems Conference (DIS '12). ACM, New York, NY, USA, 318-327. DOI: http://dx. doi .org/10.1145/2317956.2318005

31. Mario Schreiner, Roman Rädle, Hans-Christian Jetter, and Harald Reiterer. 2015. Connichiwa: A Framework for Cross-Device Web Applications. In Proceedings of the 33rd Annual ACM Conference Extended Abstracts on Human Factors in Computing Systems (CHI EA '15). ACM, New York, NY, USA, 2163-2168. DOI: http://dx.doi.org/10.1145/2702613.2732909

32. Pierre Wellner. 1991. The DigitalDesk Calculator: Tangible Manipulation on a Desk Top Display. In Proceedings of the 4th Annual ACM Symposium on User Interface Software and Technology (UIST'91). ACM, New York, NY, USA, 27-33. DOI: http://dx.doi.org/10.1145/120782.120785

33. Johannes Zagermann, Ulrike Pfeil, Roman Rädle, Hans-Christian Jetter, Clemens Klokmose, and Harald Reiterer. 2016. When Tablets Meet Tabletops: The Effect of Tabletop Size on Around-the-Table Collaboration with Personal Tablets. In Proceedings of the 2016 CHI Conference on Human Factors in Computing Systems (CHI '16). ACM, New York, NY, USA, 5470-5481. DOI : http://dx.doi.org/10.1145/2858036.2858224 